\title{
Percepciones e Intervenciones Relacionadas a la Deserción Escolar en Escuelas Oficiales de Panamá
}

\section{Nadia De León ${ }^{1}$, Defina D'Alfonso², Nyasha Warren², Manuel Dixon-Pineda ${ }^{3}$, Veyra Beckford Brown ${ }^{3}$, Jonás N. Vega García ${ }^{3}$, Ángela P. Helowicz ${ }^{3}$.}

${ }^{1}$ Centro de Investigación Educativa de Panamá, Instituto de Investigaciones Científicas y Servicios de Alta Tecnología (INDICASAT-AIP), Universidad Santa María la Antigua. El autor es Miembro del Sistema Nacional de Investigación.

${ }^{2}$ Praxis Educational Consultants

${ }^{3}$ Universidad del Istmo

*Autor para Correspondencia. E-mail: nadiadeleonporter@gmail.com

Recibido: 11 de agosto de 2020

Aceptado: 21 de septiembre de 2020

\section{Resumen}

Este estudio se basa en datos de las 345 escuelas públicas participantes en el Concurso Nacional por la Excelencia Educativa 2019, provenientes de todo Panamá. Por medio del cuestionario de inscripción se identificó el porcentaje de escuelas por región educativa y grado que identifican la deserción como un problema, refiriéndonos al abandono o ausencia de niños y jóvenes en edad escolar de la escuela. Se llevaron a cabo entrevistas en centros escolares con docentes, directores, administrativos, padres de familia y estudiantes. Por medio de un análisis cualitativo utilizando codificación por temas y análisis de frecuencias de los códigos se identificaron causas del fenómeno, estrategias para prevenirlo y para reintegrar a los estudiantes excluidos, para comprender la perspectiva de las escuelas frente a la problemática. Encontramos coherencia entre la percepción en los centros y los datos oficiales en cuanto a nivel escolar: aumenta a mayor nivel escolar. Sin embargo, las provincias que identificaron en mayor proporción que la exclusión educativa es un problema no son 
necesariamente aquellas con mayor deserción según datos oficiales. Entre las estrategias de prevención o para lograr el reintegro de estudiantes se mencionaron con mayor frecuencia comunicarse con los padres, motivar a los estudiantes, ofrecer alimentación y visitar hogares. Se encontró cierta falta de congruencia: la causa más mencionada fueron los problemas económicos (42\%), mientras que las estrategias de prevención y solución más mencionadas se asocian a los problemas familiares (33\%). Hubo poca o nula mención de dificultades académicas. En general se encontró conciencia del problema (59\%), aunque podría ser mayor dada su magnitud. Se encontraron comunidades educativas involucradas en intervenciones a nivel local, por ejemplo: un 31\% indica realizar visitas a los hogares. Los hallazgos apoyan las políticas nacionales existentes dirigidas a las barreras económicas, y sugieren otras posibles soluciones prioritarias para atender la crisis.

Palabras Clave: Exclusión educativa, matrícula educación secundaria, políticas públicas educativas, prácticas escolares, análisis cualitativo

\begin{abstract}
This study utilizes data from 345 public schools from all Panamanian provinces participating in the 2019 National Contest for Educational Excellence. The percentage of schools by educational region and grade that identify educational exclusion as a problem, meaning children and youth of school age who dropout or are absent from school, was identified utilizing the contest enrollment form. Interviews were carried out in schools with teachers, principals, administrators, parents and students. The authors identified perceptions regarding causes, strategies to prevent it and to reintegrate excluded students utilizing qualitative analysis through thematic coding and analysis of code frequencies in order to understand the perspective of the schools regarding the problem. The authors found coherence between the school's perception and the official data in terms of grade level: exclusion increased at higher grade levels. However, the provinces that identified educational exclusion as a problem in higher proportions were not necessarily those with the highest exclusion rates according to official data. Among the prevention strategies or strategies to achieve the reintegration of students, the authors most frequently identified the following were mentioned most frequently: communication with parents, motivating students, offering meals and visiting homes were mentioned. The authors found a certain lack of coungrene in that the most mentioned cause for exclusion was financial problems $(42 \%)$, while the most mentioned prevention and solution strategies were associated with family problems $(33 \%)$. There was little or no mention of academic difficulties. In general, awareness of the problem was found to be high (59\%), although it could be greater given its magnitude. Educational communities were found to be involved in interventions at the local level, for example: $31 \%$ indicated that they made visits to homes. The findings support existing national policies targeting economic barriers, and suggest other possible priority solutions to address the crisis.
\end{abstract}

Keywords: Dropout, educational exclusion, high school enrollment, perceptions, school practices 


\section{Introducción}

A pesar de haber logrado hitos importantes en cuanto al desarrollo de la educación en Panamá, como la alfabetización y la tasa de cobertura de primaria casi total, Panamá continúa enfrentando una crisis en cuanto a cobertura en la educación pre-media, que sólo alcanza a aproximadamente el 70\% de los jóvenes, y la educación media al 50\% de los jóvenes (MEDUCA, 2019). Con la mitad de nuestros jóvenes avanzando hacia convertirse en los adultos que tomarán las riendas de nuestra sociedad sin haber completado la secundaria, nos encontramos ante una catástrofe. Gran parte del problema se encuentra en la deserción escolar. Es decir, no es que estos jóvenes no fueron nunca a la escuela, sino que completaron la primaria o quizás la básica general (noveno grado) y no continuaron. Las estadísticas referentes a la deserción educativa propiciadas por el Departamento de Estadística del Ministerio de Educación (MEDUCA, 2014) indican que la tasa de deserción de estudiantes de nivel de premedia y media fue de 11,4 y es marcada la tendencia a agudizarse el problema entre el nivel primario y el secundario. Según las estadísticas de MEDUCA, la tasa de deserción inter-anual (estudiantes que no completan el año escolar) ha sido tres veces más alta en las escuelas oficiales que en las particulares en los últimos cinco años (MEDUCA, 2020). A pesar de todo esto, hacen falta estudios nacionales y públicamente accesibles que analicen las posibles causas y/o las estrategias que se promueven desde los centros educativos para prevenir y/o reintegrar estudiantes que hayan sido excluidos del sistema. Esto es de suma importancia, por la generación de este conocimiento, y porque las intervenciones efectivas para acelerar los procesos de inclusión parten de analizar la situación actual y deben tomar en cuenta las percepciones de los actores. Este estudio provee información clave para informar acerca de la problemática en el país y diseñar programas o políticas públicas que tomen en cuenta lo que las escuelas identifican como causas y las acciones y/o programas que están ejecutando.

Este estudio se basa en los cuestionarios de inscripción y entrevistas realizadas a 345 centros educativos participantes en el Concurso Nacional por la Excelencia Educativa en su edición 2019, provenientes de todo Panamá y fue realizado con el apoyo de UNICEF Panamá. Dicho concurso mide los conocimientos y competencias de los estudiantes en las áreas de Español, Matemática y Ciencias y tiene como objetivo destacar los esfuerzos de los centros educativos oficiales, por una mejor calidad de aprendizaje. El 38.35\% de los estudiantes matriculados en todo el país asisten a las escuelas que participaron durante el 2019, lo cual implica una muestra sesgada por conveniencia, pero amplia que permite la posibilidad de considerar generalizar algunas conclusiones al sistema educativo público del país. Al mismo tiempo, busca fomentar una cultura de evaluación educativa y resaltar el desempeño del docente panameño.

Los centros educativos completaron un formulario de inscripción en línea en el que se les preguntó, entre otras cosas, qué programas o acciones estaban llevando a cabo en su centro educativo para prevenir la exclusión educativa y/o lograr el reintegro de quienes abandonaron el sistema. Los cuestionarios y entrevistas utilizaron el término deserción que es el comúnmente utilizado en el país para referirnos al abandono o ausencia de niños y jóvenes en edad escolar de la escuela, aunque en el cuerpo de este artículo nos referimos a la exclusión educativa tomando activamente la posición de responsabilidad del sistema para con los niños. De las 345 escuelas inscritas el 100\% completó la encuesta y 271 también participaron de una entrevista en la cual voluntarios de diferentes organizaciones, mantuvieron un diálogo con los miembros del comité de centro conformado por padres de familia, directores, docentes, administrativos y estudiantes, para la inscripción de la escuela o colegio al concurso. En la misma se les preguntó si sentían que la exclusión educativa era un 
problema en su centro educativo y si estaban llevando adelante alguna estrategia para retener a los estudiantes. Las respuestas brindadas en ambas instancias nos permiten conocer la situación de la problemática en las escuelas oficiales de Panamá y, de forma más precisa, cuáles son las causas que identifican y las estrategias de prevención y solución que están llevando adelante.

A partir de lo anterior, este estudio se propone responder las siguientes preguntas:

1. ¿Las escuelas perciben que la problemática incide en sus centros?

2. ¿Las escuelas están llevando a cabo acciones frente a la problemática de la exclusión educativa?

3. ¿Cuáles son las causas que los centros educativos perciben como principales al fenómeno de la exclusión?

4. ¿Qué estrategias preventivas y estrategias para reintegrar estudiantes se están llevando adelante en las escuelas?

5. ¿Quiénes son los actores que intervienen con mayor frecuencia en la atención de la problemática?

6. ¿Hay coherencia entre las percepciones y las acciones en las escuelas referentes a la problemática?

\section{Antecedentes}

Este estudio entiende la exclusión educativa como el fenómeno por el cual niños y adolescentes quedan fuera de la escuela (UNICEF, 2012). Desde el 2012, UNICEF y UNESCO, impulsan la iniciativa Global por los Niños y Niñas Fuera de la Escuela, con la finalidad de construir evidencias sobre el fenómeno de la exclusión educativa e identificar estrategias de resolución que permitan la plena realización del acceso, aprendizajes de calidad y culminación de los estudios. El documento "Iniciativa Global por los Niños Fuera de la Escuela" (UNICEF, 2012) analiza la situación en la región e identifica las barreras y cuellos de botella que delimitan la exclusión y las estrategias para superarla. Afirma que 2,9 millones de niños y niñas en edad escolar primaria y 1,9 millones de adolescentes en edad de asistir a la escuela secundaria básica se encuentran excluidos en América Latina y el Caribe (UNICEF, 2012: 18).

El informe de UNICEF y UNESCO analiza diferentes casos de la región. Además, caracteriza las dimensiones de la exclusión educativa e identifica barreras de tipo económicas; socioculturales; materiales, pedagógicas y simbólicas; y políticas, financieras y técnicas. Las barreras de tipo económicas a su vez se agrupan en la insuficiencia de ingresos para la subsistencia y la dificultad para afrontar los costos de la escolarización. Las barreras socioculturales corresponden a desajustes en las representaciones sobre el derecho a la educación, concepciones familiares sobre la escuela, o el valor y prioridad que le atribuyen los padres a la escolarización de sus hijos. Las barreras materiales, pedagógicas y simbólicas se refieren a las escuelas que no cuentan con recursos materiales básicos, donde se generan o toleran procesos de segmentación que posibilitan escasas oportunidades de aprender, y escuelas con clima vincular y pedagógico deteriorado. Las barreras políticas, financieras y técnicas son atribuidas a los sistemas educativos cuya oferta es escasa, cuyo financiamiento es insuficiente o cuyas propuestas técnicas no son eficaces o pertinentes para lograr la inclusión (UNICEF, 2012). Este estudio profundiza en las económicas y las socioculturales por ser aquellas que surgieron con casi exclusiva prevalencia en las entrevistas escolares. 


\section{Metodología}

Este estudio utiliza métodos mixtos cuantitativos y cualitativos para responder a las preguntas de investigación. Para responder a la primera pregunta de investigación, se llevó a cabo un análisis cuantitativo indicando porcentajes de escuelas que identifican un problema de exclusión educativa frente a la pregunta en la entrevista "¿Siente que el tema de deserción escolar es un problema en su comunidad? ¿En su centro educativo particularmente?”. Esta información luego fue analizada según región y nivel educativo.

Para responder a la segunda pregunta de investigación se utilizó un método mixto. Frente a la pregunta del cuestionario de inscripción: "¿Qué programas llevan a cabo para evitar deserción escolar, promover la retención y/o lograr el reintegro de aquellos que han abandonado el sistema?” durante el proceso de revisión de cuestionarios, se clasificaron las respuestas que, en primer lugar, reflejaban que hacían algo al respecto y que dicha acción estaba relacionada a la problemática, o, en segundo lugar, las respuestas en blanco, las respuestas que incluían programas no relacionados a la problemática, o las que no respondían realmente a la pregunta y no permitían evaluar.

La clasificación fue llevada a cabo por dos o tres revisores (según necesidad de aclarar o no desacuerdos entre los dos primeros revisores). Para este análisis, se calculó el promedio del puntaje otorgado por los revisores del cuestionario de cada centro educativo. Se utilizaron tres categorías de acuerdo al puntaje promedio obtenido:

- 1 punto: el centro educativo sí está haciendo algo

- $\quad$ entre 0.1 y 0.99 puntos: el centro educativo quizás esté haciendo algo

- 0 puntos: el centro educativo no parece estar promoviendo acciones para evitar la deserción

Se contrastaron los datos analizados con estadísticas nacionales sobre deserción educativa del MEDUCA según nivel educativo y provincia (2014).

Para responder a la tercera, cuarta, quinta y sexta pregunta se llevó a cabo un análisis cualitativo utilizando codificación por temas y análisis de frecuencias de dichos códigos de las notas de las entrevistas llevadas a cabo en cada escuela, con el propósito de identificar causas del fenómeno y estrategias para prevenirlo, estrategias para reintegrar a los estudiantes excluidos, y comprender la perspectiva de las escuelas frente a la problemática. Para la codificación de las respuestas al cuestionario de inscripción y a las entrevistas se utilizó Atlas. ti por un grupo de codificadores después de una sesión de alineación para buscar homogeneidad en la codificación y bajo la supervisión de dos líderes. La misma se llevó a cabo siguiendo un método mixto. Se inició con códigos basados en las barreras y estrategias para superar la exclusión educativa identificadas por UNICEF y UNESCO (2012). A partir de eso, se utilizó una teoría principalmente enraizada; es decir, no se generó un mapa conceptual completo de lo que se estaba buscando a priori, sino que muchos de los códigos utilizados para clasificar las ideas presentadas emergieron de los mismos textos, y el mapa conceptual se fue creando a medida que se leyeron y analizaron los textos. Esta metodología fue preferida para poder minimizar el sesgo de los investigadores y la teoría previa, y permitir en lo posible a comunidades educativas panameñas hablar por sí mismas por medio de sus percepciones. Los codificadores utilizaron un mapa conceptual de códigos actualizado en línea y compartieron situaciones y explicación de códigos nuevos que surgían para mantener homogeneidad. Esto resultó en 112 códigos que identifican causas, estrategias preventivas, estrategias resolutivas y actores involucrados. 
Para confirmar confiabilidad entre codificadores, las codificaciones fueron revisadas por un codificador líder quién hizo las modificaciones necesarias para homogenizar cuando las hubo.

En los casos de las escuelas que completaron el cuestionario y al mismo tiempo participaron de la entrevista, se unificó el análisis del cuestionario de inscripción y de la entrevista de modo que los datos indican la presencia de cada código una sola vez basándose en si éste se había encontrado en la inscripción y/o en la entrevista de cada escuela. Finalmente, se graficó y llevó a cabo un análisis descriptivo de los resultados. Estos se interpretaron partiendo de los aportes del informe de UNICEF y UNESCO como marco teórico para caracterizar las acciones ejecutadas desde los centros educativos y la relevancia que éstas poseen en relación al fenómeno y al contexto de América Latina y el Caribe, así como los conocimientos y experiencias de los autores del sistema educativo panameño.

\section{Resultados}

Percepción sobre la presencia de un problema de exclusión educativa en el centro educativo

\section{Gráfico 1}

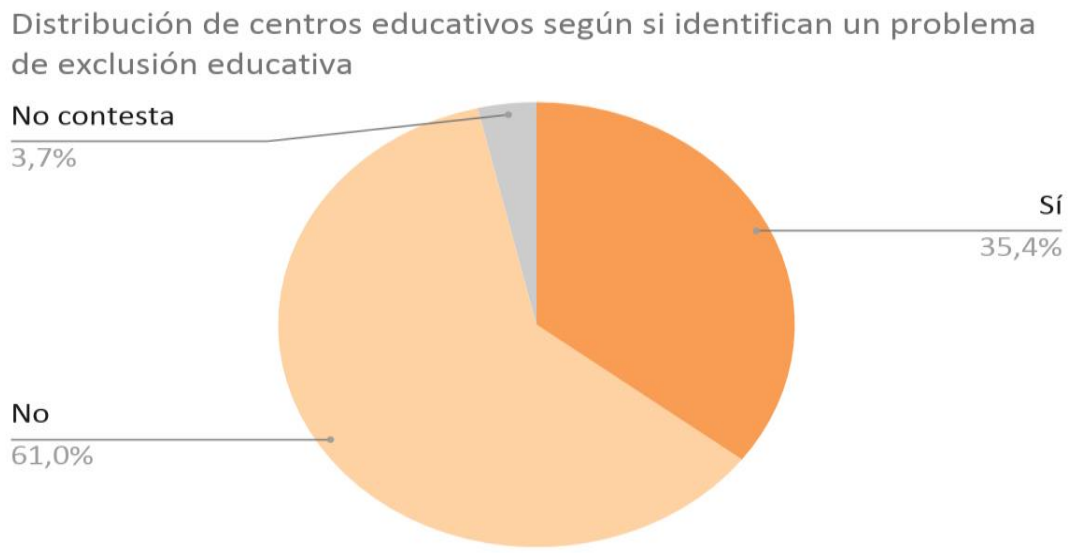

La mayoría de los centros educativos participantes de la entrevista (61\%) no identifica el fenómeno de la exclusión educativa como un problema dentro de su comunidad.

\section{Gráfico 2}

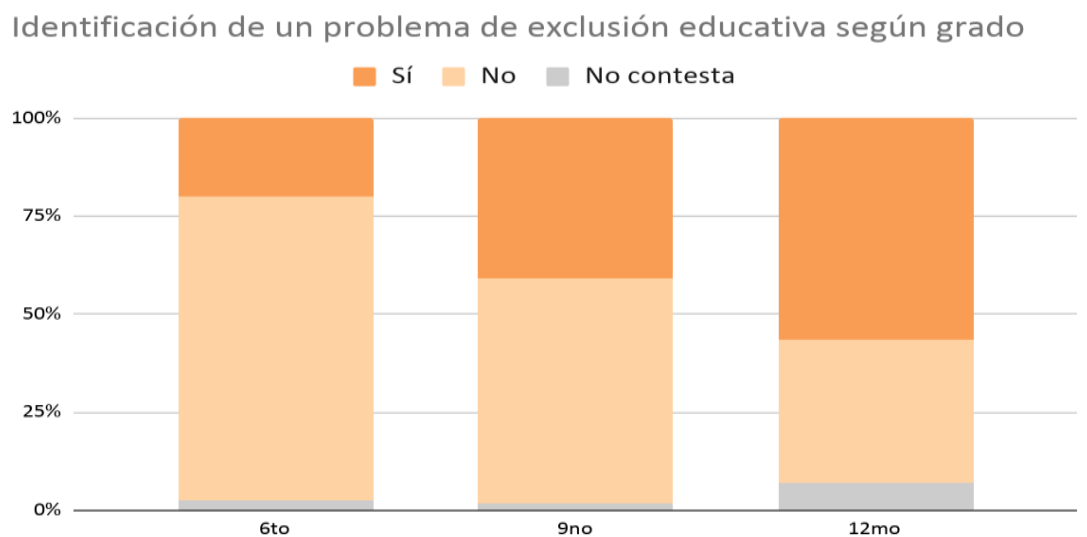


El gráfico 2 muestra que la percepción del fenómeno de la exclusión educativa se acentúa hacia 12mo. En el 2014, la tasa de deserción se comportó de manera muy similar, era menor en el nivel primario $(1,4)$ y en el nivel de premedia y media aumenta pero se mantiene igual entre ambos niveles $(11,4)$ (MEDUCA, 2014).

\section{Gráfico 3}

Porcentaje de centros que identificaron un problema de exclusión educativa por región

$100,00 \%$

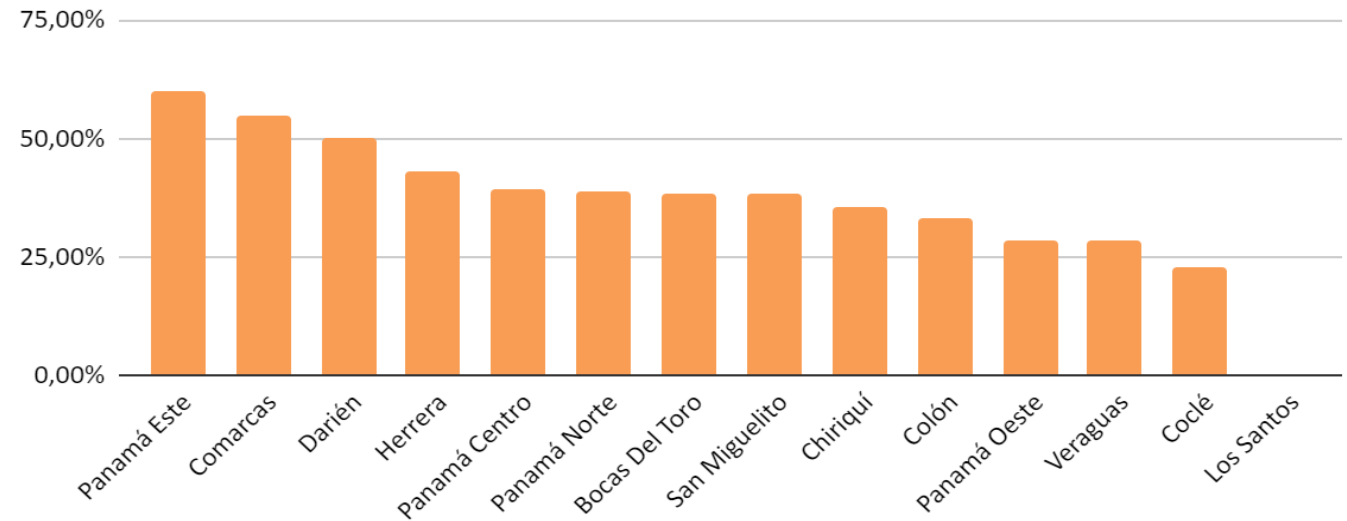

Alrededor de la mitad de las escuelas de Panamá Este, las comarcas indígenas y Darién indicaron percibir un problema de exclusión educativa en su centro; comparado a alrededor de un tercio de las escuelas de Herrera, Panamá Centro, Panamá Norte, Bocas del Toro, San Miguelito, Chiriquí y Colón; aproximadamente un cuarto de las escuelas de Panamá Oeste, Veraguas y Coclé. Ningún centro de Los Santos identificó percibir un problema de deserción en su comunidad.

Restricciones que configuran la exclusión educativa (causas)

\section{Gráfico 4}

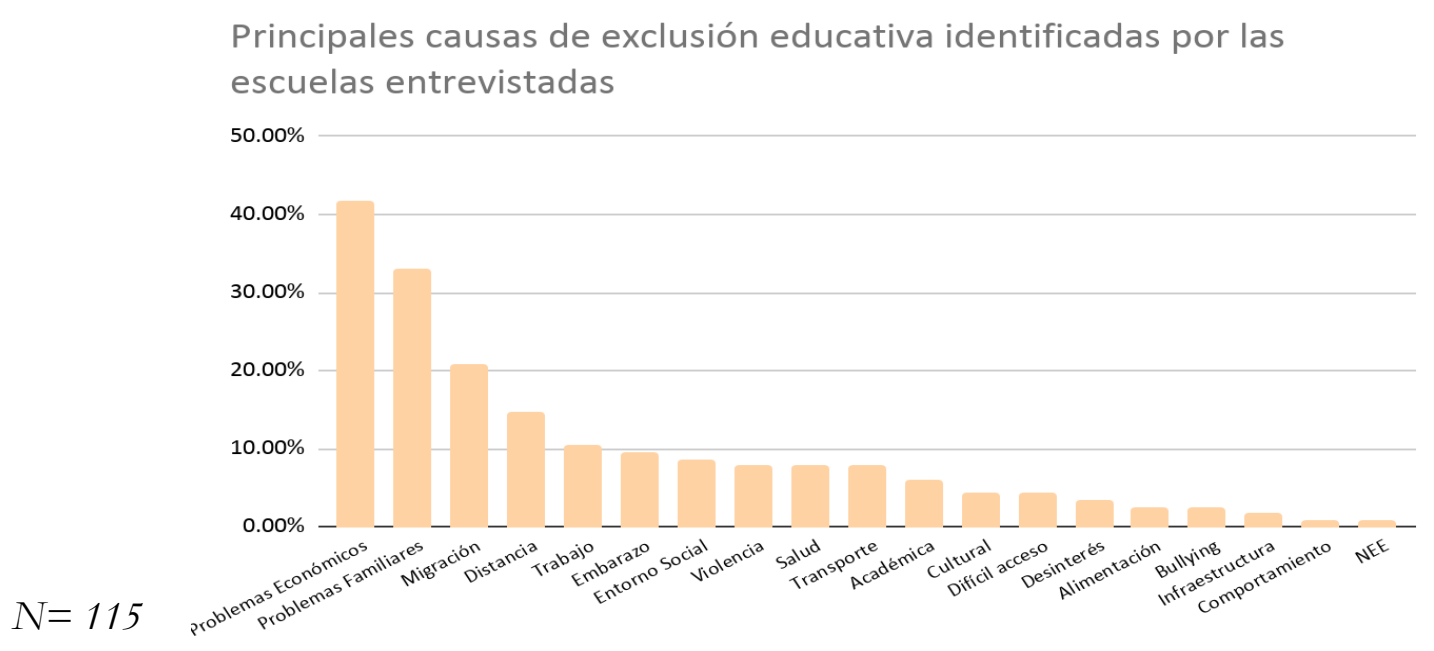


Dentro del total de centros educativos que mencionaron posibles causas (115), el 42\% sostuvo que el principal problema es de índole económico. Las escuelas mencionaron casos como: "Dentro de la investigación que se lleva a cabo con el gabinete y la trabajadora social se concluye que mucho de los estudiantes que han desertado se debe a problemas económicos", "Durante las entrevistas se trata de identificar las causas: en ocasiones es debido a falta de recursos económicos para asistir diariamente al colegio".

Por otro lado, los problemas familiares fueron la segunda causa más mencionada por las escuelas (33\%). Si bien dentro de este código abundan los casos relacionados a la desintegración familiar, nos encontramos con otros muy relacionados a minimizar el valor de asistir a la escuela. Se mencionaron casos como "hay poca o nula educación de los padres que no ven en la educación una opción para sus hijos" o "los padres que sacan a sus hijos de la escuela apenas ellos crecen para trabajar".

La migración de las familias por temas culturales y económicos también se presenta como una causa con incidencia en el contexto panameño (21\%). Las escuelas mencionaron: "El problema de deserción escolar se manifiesta entre los estudiantes de la cultura Ngöbe-Buglé que se ve en la necesidad de migrar hacia áreas en períodos de cosechas de diversos productos dentro y fuera de la provincia" o "el factor influyente principal ha sido migración de los padres a una mejora de vivienda", entre otras. En menor medida se mencionaron la distancia (15\%), problemas relacionados al transporte $(10 \%)$ y al difícil acceso (4\%).

\section{Estrategias para prevenir la exclusión educativa}

A continuación, se presentan las estrategias mencionadas por los centros educativos para prevenir la exclusión educativa de los estudiantes que se encuentran dentro de la escuela, agrupadas según las barreras.

\section{Gráfico 5}

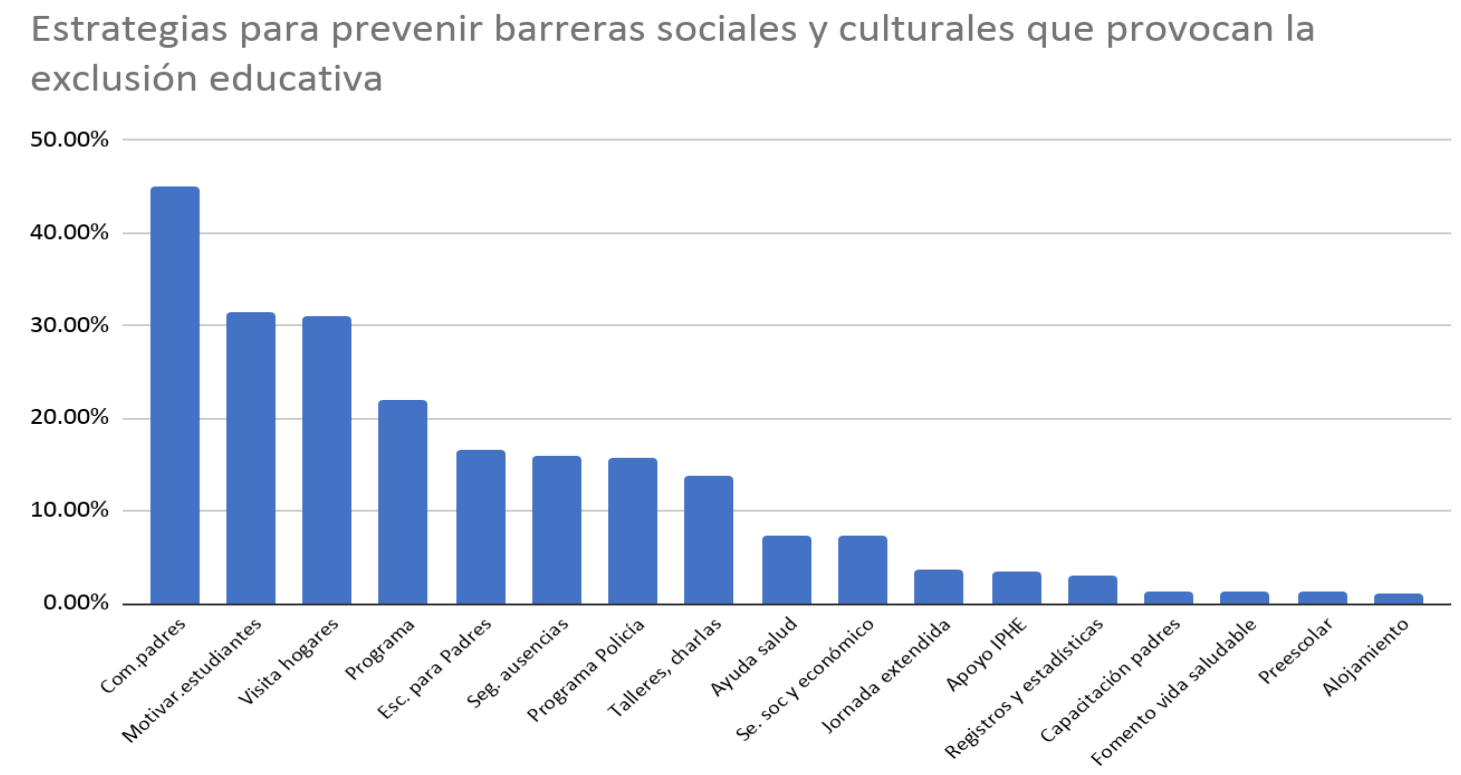

$N=297$ 
Las barreras sociales y culturales se presentaron, en mayor medida, de la mano de los problemas familiares en el contexto panameño. Del total de escuelas que mencionaron estrategias preventivas de la exclusión educativa de los estudiantes, una gran parte afirmó que sostienen una comunicación fluida con los padres de familia para prevenir que sus hijos dejen la escuela (45\%). Esta comunicación se da principalmente a partir de seguimiento de los casos en reuniones en la escuela.

Las escuelas mencionaron: "se da seguimiento a los estudiantes que falten más de 2 días seguidos, llamando a sus padres o acudientes". Otro porcentaje alto (31\%) afirmó que realizan visitas a los hogares, principalmente de los estudiantes en los que notan ausencias reiteradas para conversar con los padres y conocer la realidad del hogar. Por otro lado, un 31\% de las escuelas dijo promover acciones para motivar y orientar a sus estudiantes en sus estudios. Los centros educativos mencionaron: "las diversas actividades que se programan anualmente han hecho que los estudiantes se encuentren motivados e interesados a permanecer en las aulas escolares así como les generen las oportunidades para continuar sus estudios a nivel de pre-media, media e incluso continúen sus estudios universitarios" o "En coordinación con el Gabinete Psicopedagógico y el Departamento de Orientación, se llevan a cabo talleres de motivación para los estudiantes, con el fin de incentivar en ellos el sentido de pertenencia y hacerlos partícipes de la necesidad de la educación”.

\section{Gráfico 6}

\section{Estrategias para prevenir problemas académicos de los estudiantes que provocan la exclusión educativa}

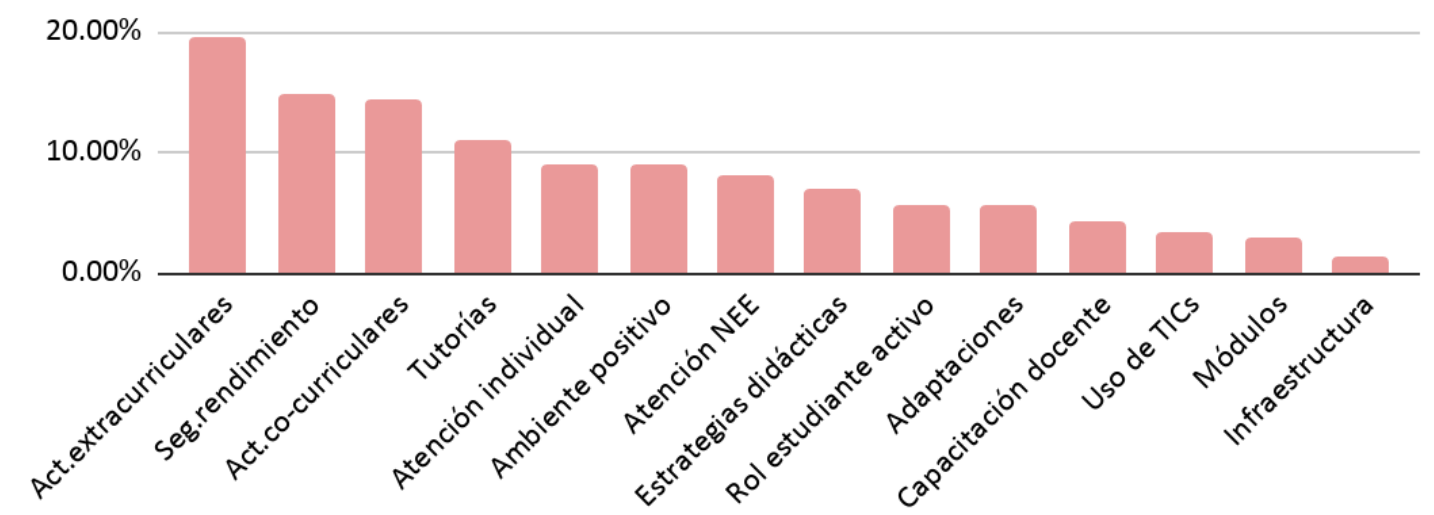

$N=297$ 
Los problemas académicos de los estudiantes fueron mencionados por apenas el $6 \%$ de las escuelas como una de las causas del fenómeno (ver gráfico 4). Sin embargo, al mencionar estrategias de prevención, hubo mayor mención de temas académicos: las escuelas mencionaron fomentar el desarrollo de actividades extracurriculares (20\%) y co-curriculares (15\%) para sus estudiantes, dar seguimiento al rendimiento de los estudiantes $(15 \%)$ y ofrecer tutorías $(12 \%)$.

\section{Gráfico 7}

\section{Estrategias relacionadas a prevenir barreras económicas que provocan la exclusión educativa}

$40.00 \%$

$30.00 \%$

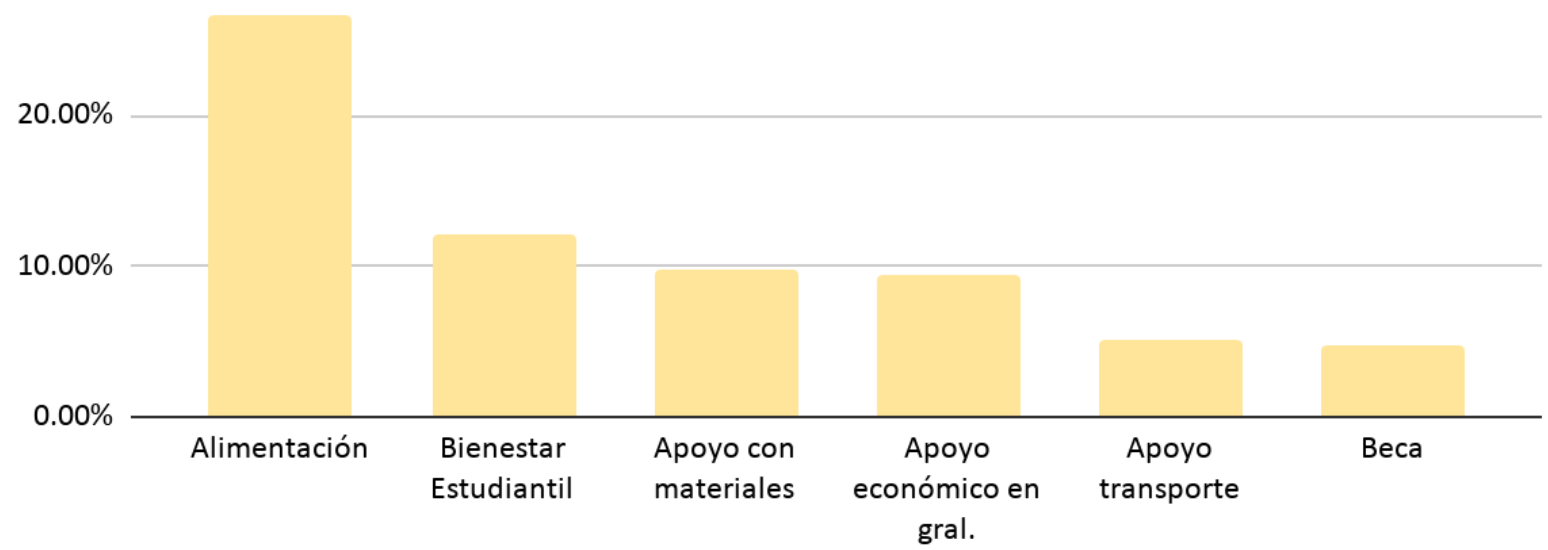

$N=297$

Las escuelas mencionaron variadas estrategias alineadas a los problemas económicos de las familias, la principal causa de exclusión educativa. Principalmente, mencionaron ofrecer alimentación en sus instalaciones (27\%): "Se autogestionan los recursos para garantizar a los estudiantes, una merienda y un almuerzo lo cual refuerza sus energías para hacer frente a la demanda académica". El 12\% de las escuelas afirmó la importancia de una comisión de bienestar estudiantil o asociación de padres de familia para prevenir la exclusión educativa a partir de la ayuda económica que brinda. El 10\% afirmó que ofrecen materiales o apoyo económico para el mismo y el $9 \%$ mencionó apoyo económico en general. Luego, el 5\% mencionaron apoyo económico para costear gastos de transporte y el otorgamiento de una beca a los estudiantes que lo necesitan.

\section{Estrategias para superar la exclusión educativa}

A continuación, se presentan las estrategias mencionadas por los centros educativos para reintegrar a los niños, niñas y adolescentes que están fuera de la escuela, agrupadas según las barreras. 


\section{Gráfico 8}

\section{Soluciones relacionadas a barreras sociales y culturales que provocan la exclusión educativa}

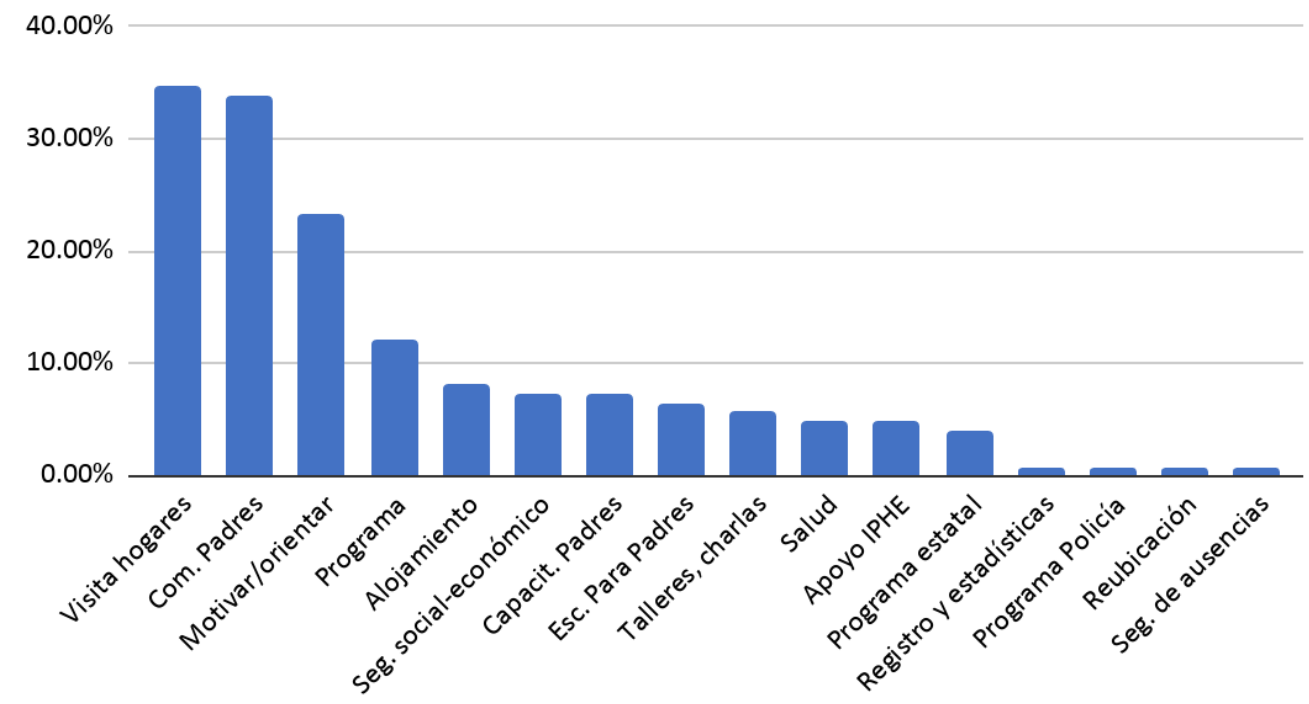

$N=124$

$\mathrm{Al}$ igual que en las estrategias preventivas, predominan las soluciones alineadas a barreras sociales y culturales. Las medidas más tomadas por las escuelas son las visitas a los hogares (35\%), comunicarse con los padres $(34 \%)$ y motivar y orientar a los estudiantes para que regresen a la escuela (23\%). Las escuelas mencionaron, "Para lograr el reintegro de aquellos que han abandonado el sistema, una actividad muy positiva ha sido la visita de la trabajadora social a los hogares de los estudiantes que presentan dificultades académicas y disciplinarias, con el propósito de conocer la causa de la deserción y brindarle el apoyo necesario para que se reintegre a las aulas".

\section{Gráfico 9}

Soluciones relacionadas a barreras económicas que provocan la exclusión educativa

$40.00 \%$

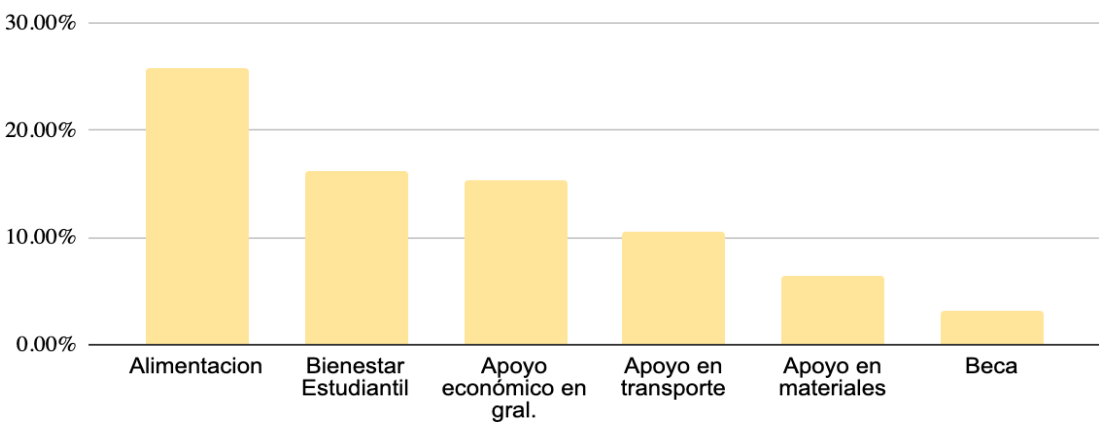


Las soluciones económicas al problema se presentaron en menor medida y, principalmente, de la mano de la alimentación que ofrece la escuela (27\%), de la Comisión de Bienestar Estudiantil (16\%), del apoyo económico en general (15\%), del apoyo económico para el transporte $(10 \%)$, para materiales escolares $(6 \%)$ y, en menor medida, el otorgamiento de una beca para lograr el reintegro de los estudiantes $(3 \%)$.

\section{Gráfico 10}

\section{Soluciones relacionadas a problemas académicos de los estudiantes que provocan la exclusión educativa $40.00 \%$}

$30.00 \%$

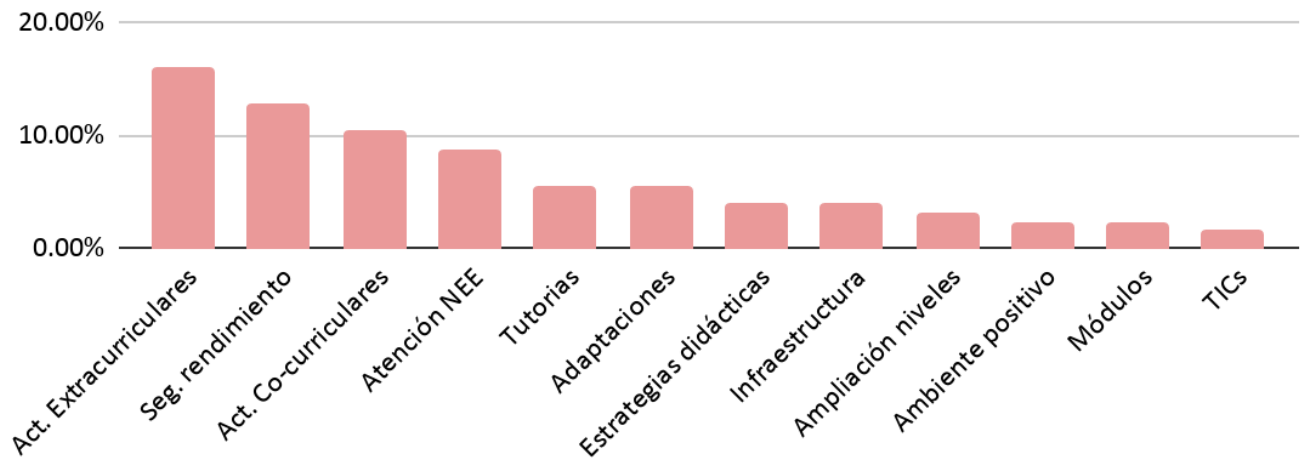

Entre las soluciones relacionadas a los problemas académicos de los estudiantes, se mencionaron con mayor frecuencia las actividades extracurriculares (16\%) y el seguimiento académico de los estudiantes $(13 \%)$. También se ejecutan actividades co-curriculares $(10 \%)$ y se brinda atención a estudiantes con necesidades educativas especiales $(9 \%)$. Algunas escuelas mencionaron, "Con relación al reintegro de los estudiantes, el departamento de orientación en conjunto con los consejeros y la administración llevamos un seguimiento con la comisión de rendimiento académico para detectar aquellos estudiantes con dificultades académicas".

Actores que intervienen para paliar el fenómeno de la exclusión educativa 


\section{Gráfico 11}

\section{Actores que intervienen para prevenir la exclusión y/o reintegrar estudiantes que abandonaron la escuela}

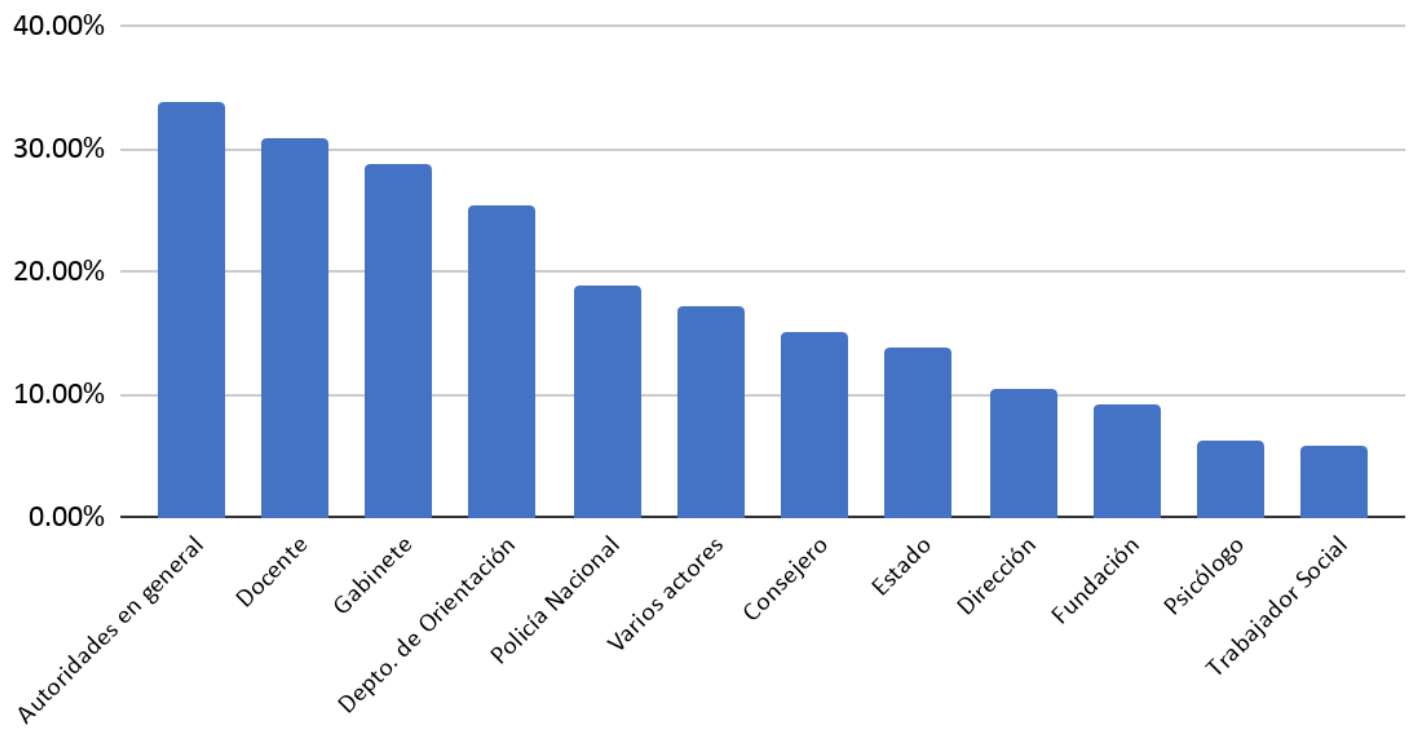

$N=239$

Entre las escuelas que mencionaron actores que intervienen con mayor frecuencia en la problemática, ya sea dándole seguimiento a partir de conversaciones con los estudiantes o padres de familia o ejecutando alguna acción o programa específico, se encuentran las autoridades en general (34\%) los docentes (31\%), el gabinete psicopedagógico (29\%), el departamento de orientación (26\%) y la Policía Nacional con sus programas DARE y GREAT (19\%). En menor medida se mencionaron la integración de varios actores, la figura del consejero, el Estado, la dirección de la escuela, fundaciones, la figura específica del psicólogo o la del trabajador social. Las escuelas mencionaron, "estudiantes que no pueden asistir por enfermedades en cama los docentes coordinan con la administración y los padres para llevarlos a su casa las clases y de esta manera no dejar la educación" o "Cuando el niño falta repetidas veces el docente investiga y luego visita el hogar para observar y dialogar con el acudiente".

\section{Conclusiones}

Como observamos en los gráficos 1 y 2 , aunque el $61 \%$ de los centros indicó no percibir un problema de deserción en sus centros; el 20\% de las escuelas primarias, el 41\% de las escuelas de pre-media, y el 56\% de las escuelas de media, identificaron tener un problema de exclusión educativa en su centro. Es el mismo patrón que hayamos en la cobertura escolar. Por ende, en cuanto a tendencias, hay coherencia entre las percepciones en los centros y los datos que documentan la realidad de la problemática. Por el otro lado, esperaríamos un porcentaje ligeramente más alto de centros de premedia y media que identificaran un problema, en contraste con los datos existentes. Es posible que la diferencia se deba a un sesgo en las escuelas que conforman la muestra, ya que son aquellas que llegaron a la segunda fase del concurso de cuyo proceso se recolectaron los datos; pero aun tomando 
esto en cuenta el nivel de la problemática en el país sugeriría una predicción de un porcentaje más alto de centros con que identifiquen enfrentarse a este reto.

Las estadísticas nos indican que las regiones que mostraron tasas más altas de deserción escolar en el nivel primario, pre-media y media fueron las comarcas indígenas, Darién, Bocas Del Toro, Coclé y Los Santos (MEDUCA, 2014). El gráfico 3 muestra que las escuelas ubicadas en Panamá Este, Comarcas, Darién y Herrera fueron las que más identificaron un problema de exclusión educativa durante la entrevista. Esto señala una conciencia apropiada del problema, primer paso para encontrar soluciones, en las comarcas indígenas y las provincias de Darién y Bocas del Toro. Los datos sugieren que Panamá Este, en general, como región, tiene una percepción de la problemática superior a su realidad. Por el otro lado, llama la atención que muy pocas escuelas de Coclé lo hicieron y ninguna de Los Santos, indicaron percibir la problemática en su centro, a pesar de estar entre las regiones con mayor exclusión según las estadísticas. Esta falta de conciencia sobre la problemática es una barrera considerable para encontrar soluciones que debe ser abordada con campañas de concienciación. De la misma manera, sería recomendable profundizar la investigación sobre el tema en la región para identificar si la problemática de deserción está mayormente restringida a algunos centros educativos con tasas extremadamente altas de deserción comparadas al resto de los centros de la región, así como las circunstancias que podrían llevar a una realidad como esa y las intervenciones particulares que podría requerir. Es claro que la temática de concientización del problema requiere intervenciones heterogéneas.

La causa principal relacionada a la exclusión educativa que identificaron las escuelas son las barreras económicas (42\%), seguido de problemas familiares (33\%), problemáticas de acceso físico (distancia, transporte, geográficas de difícil acceso) (29\%), y temáticas relacionadas a la migración (21\%). Estos resultados se alinean con las identificadas en el informe de UNICEF y UNESCO (2012), que señalan las temáticas económicas y socioculturales como las principales barreras que producen o agravan la exclusión educativa. Según UNICEF, dentro de la región latinoamericana, las barreras económicas tienen una influencia considerable en el hecho de que niños, niñas y adolescentes en edad escolar no asisten a la escuela (2012: 68). Esto implica que intervenciones que han demostrado ser eficaces en otros países latinoamericanos tienen altas probabilidades de ser efectivas también en nuestro país, como por ejemplo las transferencias económicas condicionadas (Atanasio et al, 2006; Adato, M, y Hoddinott, J., 2010; Maluccio, J. A. 2009; Galiani, S. y McEwan, P. J., 2013; García, S. y Saavedra, J. E. 2017; Herrera, L.C., 2019).

Los problemas familiares fueron la segunda causa más mencionada por las escuelas (33\%). Se relacionan a las barreras socioculturales en la demanda por la educación presentadas por UNICEF y UNESCO. Entre estas barreras se mencionan las posturas retraídas para demandar o ejercer el derecho a la educación. Por ejemplo, la escasa valoración de la educación de las familias, el escaso acompañamiento, el bajo nivel educativo de los padres, como factores influyentes en el fenómeno (UNICEF, 2012: 77). Este aspecto también estuvo presente en el contexto panameño. La naturaleza de ciclo de retroalimentación positiva intergeneracional de este fenómeno presenta un reto particular. Un posible acercamiento podría estar relacionado a la educación popular y la educación de padres de familia por medio de los programas como Escuela para Padres, dado que la participación en programas de ese tipo puede incluirse como parte de las condiciones para transferencias condicionadas. 
Las variables relacionadas a la dificultad para llegar y estar en la escuela identificadas en el estudio de UNICEF y UNESCO dentro de la dificultad para afrontar costos de escolarización (2012: 75) también fueron mencionadas por las escuelas panameñas al hablar de la distancia, los problemas relacionados al transporte y al difícil acceso. Por otro lado, mientras que la migración de las familias por temas culturales y económicos parece ser una causa con algo de incidencia en el asunto en Panamá (21\%), ésta no se destaca en los estudios analizados por UNICEF y UNESCO. Es una problemática de particular relevancia para las provincias del oeste panameño que requiere atención especial.

La mayoría de las causas mencionadas por los centros educativos se relacionan a las barreras de tipo económico y socioculturales identificadas por UNICEF y UNESCO. Aun cuando nuestra fuente de información fueron directamente las escuelas panameñas, se observa que, en el país, además, en menor medida, se identificaron barreras atribuidas a los propios centros educativos y/o al sistema educativo. En el gráfico 4 figuran el bullying (3\%), la infraestructura $(2 \%)$ y las necesidades educativas especiales $(1 \%)$ que podrían atribuirse a barreras de tipo materiales, pedagógicas y simbólicas derivadas de la escuela relacionadas al clima escolar y a las dificultades que presentan algunos centros para organizar la enseñanza en grupos diversos. Sin embargo, notablemente no se identificaron causas relacionadas al sistema educativo.

En cuanto a coherencia entre causas y soluciones encontramos cierto desfase. Mientras que en las causas identificadas por las comunidades educativas predominan las barreras de tipo económicas; las estrategias preventivas que más se mencionaron se encuentran alineadas, más bien, a la segunda causa identificada, los problemas familiares. Las estrategias de prevención de la exclusión que indican llevar a cabo las escuelas (comunicación con los padres, visitas a hogares y motivación de los estudiantes, ver gráfico 6) se alinean a la importancia de mantener una estrategia sistemática de comunicación propuesta por UNICEF y UNESCO como una estrategia que permite superar barreras socioculturales (UNICEF, 2012: 113). Por el otro lado, acorde con la problemática económica, la mayoría de las escuelas panameñas ofrece alimentación y cuenta con cierta presencia de comisiones de bienestar estudiantil y grupos de padres de familia, dos estrategias preventivas que buscan, según UNICEF y UNESCO, progresar hacia mayor justicia en recursos y posibilidades a partir de balancear ingresos familiares y suplir costos de escolarización (UNICEF, 2012: 116).

Importantemente, UNICEF y UNESCO caracterizan a las barreras que producen o agravan la exclusión como factores externos a los propios estudiantes que intervienen en el proceso, se relacionan al entorno sociocultural y económico de los estudiantes, o bien son atribuibles a las propias escuelas y o al sistema educativo. Sin embargo, muchas de las estrategias preventivas mencionadas por las escuelas panameñas se encuentran alineadas a focalizar la atención en problemas académicos de sus estudiantes o sus actitudes y valores, buscando convencerlos de permanecer por medio de la motivación. Adicionalmente, otro desfase relacionado a coherencia entre causa y soluciones identificado consiste en que únicamente el 6\% de las escuelas afirmó que el tema académico era una causa del abandono, pero se mencionaron en mucha mayor medida varias estrategias relacionadas, tales como la ejecución de actividades extracurriculares $(20 \%)$ y co-curriculares $(15 \%)$ para sus estudiantes, al seguimiento del rendimiento de los estudiantes $(15 \%)$ y tutorías $(12 \%)$.

Para los casos particulares de reinserción de estudiantes excluidos del sistema, las estrategias mencionadas por las escuelas no difieren tanto de las mencionadas en materia de prevención de la problemática, algunas pasan a mencionarse en mayor o menor medida. Por ejemplo, mientras que para 
prevenir la exclusión educativa la escuela mantiene una comunicación fluida con los padres de familia en la escuela o por teléfono, para la reinserción indican recurrir en mayor medida a la visita directa en los hogares.

UNICEF y UNESCO resaltan la importancia de que los Estados “allanen los obstáculos” que impiden a los niños y adolescentes acceder o permanecer en el camino de la educación legalmente obligatoria en cada país (UNICEF, 2012: 109). Esto indica una barrera sistémica existente pero no mencionada en el estudio: la educación obligatoria o básica general incluye sólo la pre-media, y no la media. Es justo en ese momento donde encontramos la mayor deserción. El estudio muestra que el actor con mayor presencia en el momento de la prevención y/o solución de la problemática son las autoridades del estado, e incluso figura con frecuencia inesperada la policía. Sin embargo, hay una presencia fuerte de figuras individuales, tales como el docente y los miembros del gabinete psicopedagógico o del departamento de orientación, o de la sociedad civil como grupos de padres de familia.

\section{Recomendaciones}

Los resultados nos indican la importancia de realizar estudios de este tipo, esclareciendo percepciones relevantes, prioridades particulares, y debilidades existentes. Resaltan la importancia de realizar campañas de sensibilización entre docentes y líderes educativos sobre las causas de las problemáticas de exclusión educativa, la importancia de la congruencia entre causas e intervenciones, y la literatura existente sobre intervenciones efectivas. Actores interesados en incidir en esta problemática para fortalecer esfuerzos existentes, así como diseñar e implementar nuevas acciones necesarias, han de tomar en cuenta las necesidades identificadas por las mismas comunidades educativas. Entre ellas, debe tomarse en cuenta la sistematización de la ayuda o facilitación de transporte, alimentación y materiales educativos; el fortalecimiento de programas de transferencia económica condicionada con campañas de sensibilización al público en general al respecto; la comunicación con las familias; la revisión de la relevancia curricular y de estrategias pedagógicas para el Siglo XXI; y programas de apoyo académico como tutorías. Investigaciones futuras podrían profundizar en las realidades particulares de áreas urbanas en riesgo y de zonas con temáticas de migración por agricultura, y explorar la eficacia de intervenciones actuales.

\section{Referencias}

Attanasio, O., Fitzsimons, E., Gomez, A., Lopez, D., Meghir, C., \& Mesnard, A. (2006). Child education and work choices in the presence of a conditional cash transfer programme in rural Colombia, CEPR Discussion Paper No. 5792.

Adato, M., \& Hoddinott, J. (2010). Conditional cash transfers in Latin America. Intl Food Policy Res Inst.

García, S., \& Saavedra, J. E. (2017). Educational impacts and cost-effectiveness of conditional cash transfer programs in developing countries: A meta-analysis. Review of Educational Research, 87(5), 921-965. 
Galiani, S., \& McEwan, P. J. (2013). The heterogeneous impact of conditional cash transfers. Journal of Public Economics, 103, 85-96.

Herrera, L. C. (2019). Impacto De La Beca Universal en Retención Escolar y Cumplimiento Del Ciclo Educativo. Investigación y Pensamiento Crítico, 7(1), 5-26.

Maluccio, J. A. (2009). Education and child labor: Experimental evidence from a Nicaraguan conditional cash transfer program. In Child Labor and Education in Latin America (pp. 187-204). Palgrave Macmillan, New York.

MEDUCA (2014). Estadísticas Educativas, TASA DE DESERCIÓN AÑO ESCOLAR 2014. Recuperado de http://www.meduca.gob.pa/direccion-plane/estadisticas el 30 de noviembre de 2019.

MEDUCA (2019). Estadísticas Educativas. Recuperado de http://www.meduca.gob.pa/direccionplane/estadisticas el 30 de noviembre de 2019.

MEDUCA (2020). Estudiantes Reportados como en Deserción Inter-Anual Registrado por Nivel Primario, Pre-media y Media en la República de Panamá Subsistema Formal: Año 2002-2019.

Recuperado del 2 de septiembre de 2020.

UNICEF y UNESCO (2012). Todos los niños en la escuela 2015. Iniciativa Global por los Niños Fuera de la Escuela. 